\title{
Prism adaptation with opposed base orientation: The weighting of direction information from the two eyes ${ }^{1}$
}

\author{
JOAN E. FOLEY 2 \\ UNIVERSITY OF TORONTO
}

\begin{abstract}
The aftereffect of wearing 20-diopter vertically displacing prisms with bases oriented in opposite directions on the two eyes was measured in each eye. Five groups of $20 \mathrm{Ss}$ differed in the density of a neutral filter worn over one eye. With no filter, the base-down prism dominated the directional judgments of both eyes, although there was a difference between the eyes consistent in direction with their differential treatment. The characteristics of the function relating aftereffect measures to filter density were found to depend upon the base orientation of the prism associated with the filter.
\end{abstract}

Interocular effects in prism adaptation have been explored in a limited number of conditions that provide different exposure histories for the two eyes by Foley and Miyanishi (1969). They reported complete interocular transfer following monocular exposure, confirming similar observations by Hajos and Ritter (1965) and by Pick, Hay, and Willoughby (1966). In another condition they found evidence of limited "channel-dependence," viz, in Ss who wore 20-diopter prisms over both eyes, but with their bases oriented in opposite directions (up and down), monocular aftereffects were measured in each eye in the direction to be expected for the base-down prism, but of lesser magnitude in the eye that had worn the base-up prism.

The present study includes replication of the above conditions ${ }^{3}$ and extension to the case in which the two eyes are subject to opposite vertical displacements with unequal luminance between their fields. This was achieved by the use of neutral filters of various densities, worn monocularly with ejther the base-down or the base-up prism for a given $S$.

\section{METHOD}

Subjects

Forty male and 40 female undergraduates participated in the experiment. Most Ss were freshmen aged 19-20 years. None was familiar with prism adaptation phenomena.

\section{Apparatus}

Two 20-diopter wedge prisms were mounted on as opaque face in a modified diving mask. The prisms could be rotated in their mounts to any desired base orientation. The visual field of each eye was circular and measured about $70 \mathrm{deg}$. Inside the mask, brackets were provided to hold neutral density filters over either eye, as desired. Three glass-mounted Kodak Wratten filters, of ND .7, 1.3, and 1.9, respectively, were available. These correspond to transmission factors of 2 , .05 , and .0125 . An opaque cover (ND $\infty$, Transmission 0) was also available.

The test apparatus comprised a vertical array of dim $42-\mathrm{ft}$ lights, $50 \mathrm{~cm}$ ahead of the $S$, whose head was positioned by means of a chin rest and bite bar. Removable shields permitted monocular presentation of the test lights. The apparent height of the target light, viewed in darkness, was indicated by the $S$, who reached forward with his right hand and made a mark with a felt pen on a sheet of paper to the right of the display. The marking sheet could not be seen by the $S$.

\section{Procedure}

Following 12 practice judgments, the S was pretested. Most judgments were of a target (T) approximately $81 / 2$ deg below the horizon, but "dummies" randomly selected from two higher and two lower targets within $6 \mathrm{deg}$ of the central one were also presented. Dummy targets were presented as a precaution against stereotyped arm movement within or between test sessions. In particular, such stereotyping could obscure interocular differences where rightand left-eye tests are mixed. Twelve trials were given at pretest and at each subsequent test session. A table of random permutations was used on each occasion to intermix four $T$ and two dummy presentations to each eye. Only $T$ judgments were analyzed.

After pretesting, the $S$ was fitted with a pair of goggles appropriate to the treatment group to which he had been allocated. He then spent $10 \mathrm{~min}$ in the company of the $E$ and another $S$ walking along corridors in the building. Overhead lights provided fairly even illumination at about $22 \mathrm{fc}$. He was then tested (without goggles). The goggles were refitted and another $10 \mathrm{~min}$ was spent walking. A final test without goggles concluded the session.

\section{Design}

Eight male and eight female $S s$ were allocated to each of the five filter conditions: ND $0, .7,1.3,1.9$, and $\infty$. Half the Ss of each sex wore the filter in conjunction with the base-up prism and half with the base-down. In each of these subgroups, half the $\mathrm{Ss}$ wore the base-up prism over the right eye and half wore it over the left eye. That is, two males and two females were run in each of the 20 combinations of filter density, filter arrangement (with respect to base direction), and base arrangement (with respect to eye). In the case of Ss not wearing a filter (ND 0), the filter arrangement condition has no meaning. For purposes of analysis only, the group was treated as including two subgroups with different filter arrangements. The allocation of the individuals was arbitrarily decided before the experiment.

Within-S variables in the experiment were exposure duration (tests were given at 10 and $20 \mathrm{~min}$ ) and eye tested (whether its prism base was up or down).

\section{RESULTS}

Performance on the $t$ tests at 10 and $20 \mathrm{~min}$ was expressed as an angular shift from pretest performance for a given eye in each $S$ (aftereffect). An upward $(+)$ shift would be adaptive for the eye wearing the base-up prism and a downward shift for that wearing the base-down prism. Complete compensation demands a shift of about $11 \mathrm{deg}$. The effects on performance of filter density, filter arrangement, and eye tested are shown in Fig. 1. These represent mean monocular aftereffects in angular units collapsed over the variables of base arrangement and exposure duration. Analysis of variance showed no reliable effects associated with base arrangement $[F(1,60)=.18]$, but the main effect of duration $[F(1,60)=8.2, p<.01]$ reflects a tendency for markings made at the second test to be lower down than those made at the first. A Base Arrangerment by Duration interaction $[F(1,60)=4.00, p=.05]$ indicates that the duration effect is smaller when the base-down prism is on the right eye. These effects are of trivial interest in the context of this study.

The main effects of filter density $[F(4,60)=2.84, \quad p<.05]$, filter arrangement $[F(1,60)=30.52, p<.001]$, and eye tested $[F(1,60)=4.00, p=.05]$ reach acceptable levels of statistical significance. Of more interest, however, are 


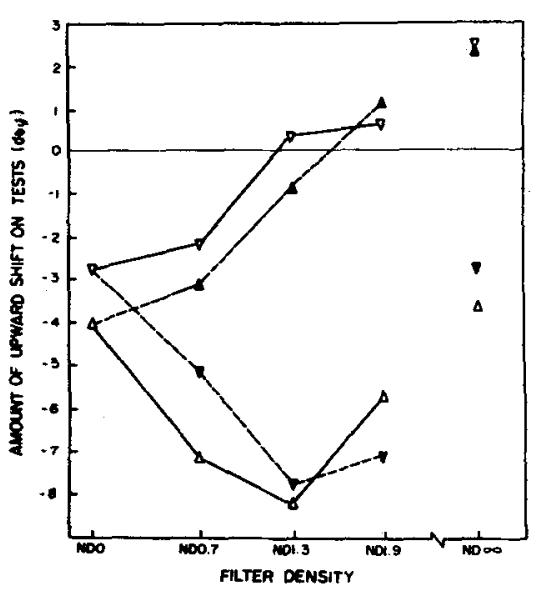

Fig. 1. Magnitude and direction of aftereffect measured in a given eye as a function of the density of a neutral filter worn on one eye during binocular exposure. Base orientation and filter treatment during exposure of the eye tested are indicated by the orientation and color of triangles on the graph, e.g: $\nabla$ measurement from eye which was exposed to base-up prism without filter; $\mathbb{M}$ measurement from eye wearing base-down prism with filter ( $\nabla$ and $\Delta$ are measured in the same $S s$ ).

certain interactions among these variables.

As expected on the basis of Foley and Miyanishi's results, Fig. 1 indicates that the difference between the base-up and base-down eye observed in the ND 0 group tends to diminish as the density of filter worn over either eye increases to $\infty$. In the analysis, however, the Density by Eye Term falls short of the .05 level of confidence $[F(4,60)=2.06$, requires 2.52]. Interocular transfer at ND $\infty$ in the present study appears to be incomplete.

The other important interaction is that of Density by Filter Arrangement $[F(4,60)=3.26, p<.05]$. Figure 1 shows that this interaction takes an unexpectedly complex form. Although the transition from ND 0 to ND $\infty$ is apparently simple when the field of the base-down prism is dimmed, such is not the case when the filter is worn in conjunction with the base-up prism. Post-hoc trend analyses were applied to each of the four functions of density shown in Fig. 1. For these purposes, the levels of density were treated as being equally spaced. When the field of the base-down prism is dimmed, the functions for both eyes show strong linear components [base-up $F(1,35)=9.36$, $p<.01$; base-down $F(1,35)=18.70$, $\mathrm{p}<.001]$. Quadratic and other trends fail to reach significance. However, when the field of the base-up prism is dimmed, the functions for both eyes show nonsignificant linear components. The quadratic term satisfies the .01 level of confidence in both cases [base-up $F(1,35)=8.26 ; \quad$ b a s e-down $\mathrm{F}(1,35)=7.55]$.

Only one other effect in the analysis met the .05 confidence level, namely, the Filter A rrangement by Duration interaction $[F(1,60)=4.00, p=.05]$. The downward drift in markings from first to second test noted earlier is smaller when the field of the base-up filter is the one that is dimmed. Larger negative scores are obtained with this filter arrangement regardless of exposure duration, since the base-down exposure produces downward shifts from pre- to posttest. This interaction therefore appears to be best characterized as a "negative ceiling" effect and is of minor interest in the present context.

\section{DISCUSSION}

The overriding influence of the base-down prism orientation in the ND 0 condition, which was noted by Foley and Miyanishi, is confirmed by the present study. The inclusion of the base arrangement control eliminates the possibility that this effect can be attributed to some advantage enjoyed by the left eye per se (all of Foley and Miyanishi's Ss wore the base-down prism on the left eye) Neither does it appear to represent a general advantage of upward displacement in the production of aftereffects, since those measured with different base orientations after monocular exposure $(\mathrm{ND} \infty)$ are approximately symmetrical about 0-deg shift (see Fig. 1). That is, there would seem to be a genuine preference for one set of information when conflicting information about spatial orientation is carried over two input channels. It could be hypothesized that this preference reflects the usefulness of the information received from the respective channels for the execution of the task to be performed during the prism exposure. In this case, the task is walking, and the field of the eye wearing the base-down prism includes more floor and less ceiling for any given position of the head than that of the eye wearing the base-up prism. The present study, however, cannot separate this possibility from others. For example, an object viewed through the base-down prism is imaged higher than when viewed through the base-up prism and, in the relatively complex visual display presented by the hallways, important specific superpositions might very well be involved (people seen walking through the ceiling lights, etc.). Further, the test targets were always below the horizon. During exposure, displacement in the lower half of the field is greater with the base-down prism.
Gaze-contingent effects have been described by Kohler (1964) and by Pick and Hay (1966).

Although a preference for one set of information is evidenced, the existence of the other is clearly recognized. There is a difference in test performance between the eyes that is consistent in its direction with their differential exposure histories, again confirming Foley and Miyanishi. The disappearance of the difference with an increase in filter density was not as clear-cut as was anticipated. It remains to be established whether or not the apparent lack of complete interocular transfer in the ND $\infty$ condition is related to the use of an opaque occluder in place of the diffusing material employed by Foley and Miyanishi. There are two reasons why it seems more likely that it is attributable to a sampling variation: (1) The difference is actually reversed in the ND 1.9 condition, and (2) Hajos and Ritter (1965) obtained complete transfer while using opaque occluders.

Consideration of the ND 0 and ND $\infty$ conditions suggests a model of weighted averaging in spatial judgment where weights assigned depend upon the relative utility of information available to either eye and upon the eye being used in testing. The results for intermediate densities when the field of the eye wearing the base-down prism is dimmed tend to support such a model. As the luminance of the "preferred" field is decreased, there is an apparent decrease in the weight assigned to this information, resulting in a reversal in the direction of the aftereffect. This will not suffice, however, in the case where the luminance of the "nonpreferred" field is decreased. Such a model would lead to an expectation of a monotonic function of negative slope. The obtained result, where aftereffects of approximately the same order of magnitude are obtained at ND 0 and ND $\infty$ and maximum negative scores at an intermediate filter density, suggests a system that is more inclined to "bet" on the preferred field when the other is dimly visible than when it is not given at all. This feature of performance emphasizes the importance of conceptualizing the situation as one in which there may be a selective weighting of available altematives. It precludes, however, the application of the relatively simple model at first suggested.

The interocular effects described here can be construed as compatible with any of a variety of extant theories of prism adaptation. Webster (1969), for example, has referred to cognitive, motor-kinaesthetic, and oculomotor mechanisms in adaptation. Studies of the type reported here potentially lead to a 
definition of those characteristics of the optical array that are important in controlling the adaptation process and contribute to our understanding of how binocular visual input is utilized in spatial judgments.

\section{REFERENCES}

FOLEY, J. E., \& MIY ANISHI, K. Interocular effects in prism adaptation. Science, 1969 , 165,311 .

HAJOS, A., \& RITTER, M. Interocular effects of adaptation to prismatic spectacles. Acta Psychologica, 1965, 24, 81-90.

KOHLER, I. The formation and transformation of the perceptual world. Psychological Issues $1964,3,1-173$.

PICK, H. L., \& HAY, J. C. Gaze-contingent adaptation to prismatic spectacles. American Journal of Psychology, 1966, 79, 443-450.

PICK, H. L., HAY, J. C., \& WILLOUGHBY, R. H. Interocular transfer of adaptation to prismatic distortion. Perceptual \& Motor Skills, 1966, 23, 131-135.

WEBSTER, R. G. The relationship between cognitive, motor-kinaesthetic, and oculomotor adaptation. Perception \& Psychophysics, 1969 $6,33-38$.

\section{NOTES}

1. This study was supported by the National Research Council of Canada, Grant No. APA-1. 2. Address: University of Toronto, Toronto, Ontario, Canada.

3. Foley and Miyanishi used a diffusing material in the transfer condition, while an opaque cover was used in the present study. 